PROCEEDINGS OF THE

AMERICAN MATHEMATICAL SOCIETY

Volume 125, Number 12, December 1997, Pages $3747-3752$

S 0002-9939(97)04069-0

\title{
EQUIVARIANT ACYCLIC MAPS
}

\author{
AMIYA MUKHERJEE AND ANIRUDDHA C. NAOLEKAR
}

(Communicated by Thomas Goodwillie)

\begin{abstract}
In this paper we apply a recently developed new version of the Bredon-Illman cohomology theory to obtain an equivariant analogue of a result of Kan and Thurston, which implies that a connected CW-complex has the homotopy type of a space obtained by applying the plus construction of Quillen to certain Eilenberg-Mac Lane spaces.
\end{abstract}

\section{Statement of Results}

A space $X$ is acyclic if its reduced integral homology $\widetilde{H}_{*}(X)=0$. The universal coefficient theorem then implies that $X$ is acyclic if and only if the reduced cohomology $\widetilde{H}^{*}(X ; G)=0$ for every coefficient group $G$. Also a map $f: X \longrightarrow Y$ is acyclic if its homotopy fibre is acyclic. We say that a $G$-space $X$ is $G$-acyclic if its reduced Bredon-Illman cohomology $\widetilde{H}_{G}^{*}(X ; \lambda)=0$ for every abelian $O_{G}$-group $\lambda$, and a $G$-map $f: X \longrightarrow Y$ is $G$-acyclic if its $G$-homotopy fibre is $G$-acyclic.

Here $O_{G}$ denotes the category of orbit spaces $G / H$ and $G$-maps, and an $O_{G^{-}}$ group is a contravariant functor $O_{G} \longrightarrow$ Grp. Other notions like $O_{G}$-space, $O_{G}$-fibration, etc. have similar meaning (terminology depending on the nature of codomain of the functors). The homotopy $O_{G}$-group $\underline{\pi}_{n}(X)$ of a $G$-space $X$ with a stationary point $x^{0} \in X^{G}$ as base point is defined by $\underline{\pi}_{n}(X)(G / H)=\pi_{n}\left(X^{H}, x^{0}\right)$ and $\underline{\pi}_{n}(X)(\widehat{g})=\pi_{n}(g)$, where $\widehat{g}: G / H \longrightarrow G / K$ is a morphism in $O_{G}$ arising from a subconjugacy relation $g^{-1} H g \subseteq K$, and $g: X^{K} \longrightarrow X^{H}$ is the left translation by $g$. A $G$-map $f: X \longrightarrow Y$ induces a morphism of $O_{G}$-groups $\underline{\pi}_{n}(f): \underline{\pi}_{n}(X) \longrightarrow \underline{\pi}_{n}(Y)$ defined by $\underline{\pi}_{n}(f)(G / H)=\pi_{n}\left(f^{H}\right)$, where $f^{H}=f \mid X^{H}$.

Given an $O_{G}$-group $\lambda$ (where $G$ is a compact Lie group) and an integer $n \geq 1$, there is a $G$-space $X$ such that $\underline{\pi}_{n}(X)=\lambda$ and $\underline{\pi}_{j}(X)=\underline{0}$ if $j \neq n$. This $G$-space is the classifying space for the Bredon-Illman cohomology, and is called an equivariant Eilenberg-Mac Lane space $K(\lambda, n)$ of type $(\lambda, n)$ (see [5]).

For a $G$-space $X$, there is a concept of an equivariant local coefficients system $M$ on $X$, and also of an equivariant cohomology $H_{G}^{*}(X ; M)$ (see [8]). This cohomology reduces to the equivariant singular cohomology of Bredon and Illman [2], [7] when $M$ is simple in a certain sense, and to the Steenrod cohomology with the classical local coefficients system when $G$ is trivial. In Section 2 we present an alternative description of $H_{G}^{*}(X ; M)$ in a way which is best suited in the context of $G$-acyclicity.

Received by the editors October 16, 1995 and, in revised form, July 19, 1996.

1991 Mathematics Subject Classification. Primary 55N25, 55N91.

Key words and phrases. Equivariant cohomology, $G$-acyclic map, $G$-homotopy equivalence.

(c)1997 American Mathematical Society 
Now suppose that $G$ is finite, and consider $G$-spaces $X$ which are compactly generated weakly Hausdorff with base point $x^{0} \in X^{G}$ such that $X$ has the $G$-homotopy type of a $G$-connected $G$-CW-complex. Then, in line of Kan and Thurston [6], our first main theorem is

Theorem 1.1. For a $G$-space $X$, there exist an $O_{G}$-group $\lambda$ with a perfect normal $O_{G}$-subgroup $\eta$ and a $G$-acyclic map

$$
f: K(\lambda, 1) \longrightarrow X
$$

which is natural with respect to $X$, such that $\operatorname{Ker} \underline{\pi}_{1}(f)=\eta$, and

$$
f^{*}: H_{G}^{*}(X ; M) \longrightarrow H_{G}^{*}\left(K(\lambda, 1) ; f^{*} M\right)
$$

is an isomorphism for every equivariant local coefficients system $M$ on $X$.

Given a $G$-space $X$ and a perfect normal $O_{G}$-subgroup $\eta$ of $\underline{\pi}_{1}(X)$, it is possible to construct a $G$-space $X_{\eta}^{+}$by applying the plus construction of Quillen [9] to each $X^{H}$ with respect to the group $\eta(G / H)$, and then combining the resulting spaces together by means of a functorial bar construction. It turns out that the $G$-space $X_{\eta}^{+}$is completely determined by the pair $\left(\underline{\pi}_{1}(X), \eta\right)$ up to $G$-homotopy equivalence. More specifically, we have the following two theorems which provide a classification of $G$-acyclic maps from a given $G$-space.

Theorem 1.2. If $X$ is a $G$-space and $\eta$ a perfect normal $O_{G}$-subgroup of $\underline{\pi}_{1}(X)$, then there exist a G-space $X_{\eta}^{+}$and a G-acyclic map $f: X \longrightarrow X_{\eta}^{+}$such that $\operatorname{Ker} \underline{\pi}_{1}(f)=\eta$.

Theorem 1.3. If $f: X \longrightarrow Y$ and $f^{\prime}: X \longrightarrow Y^{\prime}$ are $G$-maps, where $f$ is $G$-acyclic, then there is a $G$-map $h: Y \longrightarrow Y^{\prime}$ with $h f \simeq_{G} f^{\prime}$ if and only if $\operatorname{Ker} \underline{\pi}_{1}(f) \subseteq \operatorname{Ker} \underline{\pi}_{1}\left(f^{\prime}\right)$; moreover, any two such $h$ are $G$-homotopic. In addition, if $f^{\prime}$ is $G$-acyclic, then $h$ is also $G$-acyclic, and $h$ is a G-homotopy equivalence if and only if $\operatorname{Ker} \underline{\pi}_{1}(f)=\operatorname{Ker} \underline{\pi}_{1}\left(f^{\prime}\right)$.

Finally, we obtain as an application our second main theorem which is

Theorem 1.4. Given a $G$-space $X$, there exists an $O_{G}$-group $\lambda$ with a perfect normal $O_{G}$-subgroup $\eta$ such that $X$ has the $G$-homotopy type of $K(\lambda, 1)_{\eta}^{+}$.

We note that the condition of $G$-connectivity of $X$ is a necessary condition for each of the main theorems to be true, and therefore cannot be avoided.

The proofs of the theorems appear in Section 3.

\section{CRIteria FOR $G$-ACYClicity}

The proofs of our theorems are based on the following two propositions. The first implies that a $G$-map $f: X \longrightarrow Y$ is $G$-acyclic if and only if each $f^{H}: X^{H} \longrightarrow Y^{H}$ is acyclic, and then the second gives the cohomological assertion of Theorem 1.1.

Proposition 2.1. A G-space $X$ is $G$-acyclic if and only if each $X^{H}$ is acyclic.

Proposition 2.2. If a G-map $f: X \longrightarrow Y$ is $G$-acyclic, then $f$ induces an isomorphism

$$
f^{*}: H_{G}^{*}(Y ; M) \longrightarrow H_{G}^{*}\left(X ; f^{*} M\right)
$$

for every equivariant local coefficients system $M$ on $Y$. 
Proof of Proposition 2.1. There is a spectral sequence

$$
E_{2}^{p, q}=\operatorname{Ext}^{p}\left(\underline{\widetilde{H}}_{q}(X), \lambda\right) \Longrightarrow \widetilde{H}_{G}^{p+q}(X ; \lambda),
$$

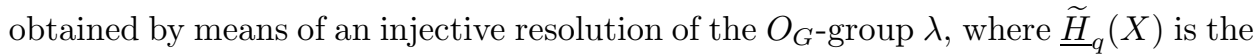

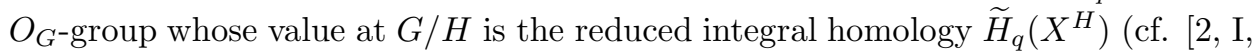
$\S 10])$. Since the category of abelian $O_{G}$-groups has sufficiently many injectives, we can embed the $O_{G}$-group $\underline{\widetilde{H}}_{q}(X)$ in an injective $O_{G}$-group $\lambda_{q}$. Then, we have in the corresponding spectral sequence $E_{2}^{p, q}=0$ for $p>0$. Therefore, if $X$ is $G$-acyclic, then

$$
0=\widetilde{H}_{G}^{q}\left(X, \lambda_{q}\right) \cong \operatorname{Ext}^{0}\left(\underline{\widetilde{H}}_{q}(X), \lambda_{q}\right)=\operatorname{Hom}\left(\underline{\widetilde{H}}_{q}(X), \lambda_{q}\right) .
$$

This implies that $\underline{\widetilde{H}}_{q}(X)=0$ as we have already a monomorphism $\underline{\widetilde{H}}_{q}(X) \longrightarrow \lambda_{q}$. Since this happens for every $q$, each $X^{H}$ is acyclic.

The converse follows easily again from the same spectral sequence. This completes the proof.

Turning now to Proposition 2.2, let us recall briefly from [8, §8] an alternative description of the equivariant cohomology $H_{G}^{*}(X ; M)$.

First note that an equivariant local coefficients system $M$ on $X$ is a contravariant functor $M: \Pi X \longrightarrow \mathbf{A b}$, where $\Pi X$ is the following category. An object of $\Pi X$ is a $G$-map $x_{H}: G / H \longrightarrow X$, and a morphism $[\widehat{g}, \phi]: x_{H} \longrightarrow y_{K}$ is a certain equivalence class of pairs $(\widehat{g}, \phi)$, where $\widehat{g}: G / H \longrightarrow G / K, g^{-1} H g \subseteq K$, is a $G$-map, and $\phi: G / H \times I \longrightarrow X$ is a $G$-homotopy from $x_{H}$ to $y_{K} \circ \widehat{g}$.

Given $M$, we define an $O_{G}$-group $M_{0}: O_{G} \longrightarrow$ Ab by sending $G / H$ to $M\left(x_{H}^{0}\right)$, and sending a $G$-map $\widehat{g}: G / H \longrightarrow G / K$ to $M([\widehat{g}, k])$, where $x_{H}^{0}$ is an object in $\Pi X$ given by the constant $G$-map $G / H \longrightarrow x^{0} \in X$, and $[\widehat{g}, k]: x_{H}^{0} \longrightarrow x_{K}^{0}$ is a morphism in $\Pi X$ given by the constant homotopy $k$ on $x^{0}$. Note that the bijection $b: X^{H} \longrightarrow \operatorname{Map}_{G}(G / H, X), b(x)(g H)=g x$, is implicit in the definition. In fact, this makes $M_{0}$ a $\underline{\pi}_{1}(X)$-module with action $\rho: \underline{\pi}_{1}(X) \times M_{0} \longrightarrow M_{0}$ given by $\rho(G / H)(\alpha, m)=\bar{M}(b(\alpha))(m)$, where $\alpha \in \pi_{1}\left(X^{H}, x^{0}\right)$ and $b(\alpha): x_{H}^{0} \longrightarrow x_{H}^{0}$ is an equivalence in $\Pi X$.

Next, consider the family of universal covering spaces $p_{H}: \widetilde{X}^{H} \longrightarrow X^{H}, H \subseteq G$. Then, for a $G$-map $\widehat{g}: G / H \longrightarrow G / K$, the left translation $g: X^{K} \longrightarrow X^{H}$ lifts to a map $\widetilde{g}: \widetilde{X}^{K}, \longrightarrow \widetilde{X}^{H}$ which is unique up to the choice of base points over $x^{0}$ in $\widetilde{X}^{K}$ and $\tilde{X}^{H}$.

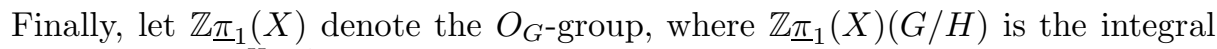
group ring $\mathbb{Z} \pi_{1}\left(X^{H}, x^{0}\right)$.

Then the cohomology $H_{G}^{*}(X ; M)$ for a finite group $G$ may be obtained by means of a cochain complex $S_{\pi, G}^{*}\left(\mathcal{U} ; M_{0}\right)$, where $\mathcal{U}$ is what we call the universal $O_{G^{-}}$ covering space of $X$. The $n$th group $S_{\pi, G}^{n}\left(\mathcal{U} ; M_{0}\right)$ of this cochain complex is a subgroup of

$$
\bigoplus_{H \subseteq G} \operatorname{Hom}_{\mathbb{Z} \underline{\pi}_{1}(X)(G / H)}\left(C_{n}\left(\widetilde{X}^{H}\right), M_{0}(G / H)\right)
$$

consisting of elements $c=\left\{c_{H}\right\}_{H \subseteq G}$ which satisfy the condition : if two equivariant singular $n$-simplexes $\sigma: \triangle_{n} \longrightarrow \widetilde{\widetilde{X}}^{H}$ and $\tau: \triangle_{n} \longrightarrow \widetilde{X}^{K}$ are connected by a $G$-map $\widehat{g}: G / H \longrightarrow G / K$ such that $\sigma=\widetilde{g} \circ \tau$, then $M_{0}(\widehat{g})\left(c_{K}(\tau)\right)=c_{H}(\sigma)$. Note that the condition is a simplified version of a general case where $G$ is a compact Lie group (see $[8,(8.3)])$. 
The following definitions and notations are preparatory to our next lemma which provides yet another description of $H_{G}^{*}(X ; M)$.

Let $L$ be a right $\underline{\pi}_{1}(X)$-module which acts on $M_{0}$ with actions $\theta: L \times \underline{\pi}_{1}(X) \longrightarrow L$ and $\omega: L \times M_{0} \longrightarrow M_{0}$ such that $\omega \circ(\theta \times i d)=\omega \circ(i d \times \rho)$.

Here are two examples of $L$ which will be important in the proof of Proposition 2.2 .

Example 2.3. Take $L=\underline{\pi}_{1}(X), \omega=\rho$ as defined above, and $\theta=$ multiplication.

Example 2.4. Let $f: X \longrightarrow Y$ be a $G$-map and $M$ an equivariant local coefficients system on $Y$. Then $\left(f^{*} M\right)_{0}=M_{0}$. Take $L=\underline{\pi}_{1}(Y)$, and $\theta: \underline{\pi}_{1}(Y) \times \underline{\pi}_{1}(X) \longrightarrow$ $\underline{\pi}_{1}(Y)$ as $\theta(G / H)(\beta, \alpha)=\beta \cdot f_{*}^{H}(\alpha)$. Let $\omega: \underline{\pi}_{1}(Y) \times M_{0} \longrightarrow M_{0}$ be as in Example 2.3 , and $\rho: \underline{\pi}_{1}(X) \times M_{0} \longrightarrow M_{0}$ be given by $\rho(G / H)(\alpha, m)=\omega(G / H)\left(f_{*}^{H}(\alpha), m\right)$.

We shall denote the $L$ of this example by $f^{*} \underline{\pi}_{1}(Y)$.

Consider the $O_{G}$-group $\underline{C}_{n}(X ; L): O_{G} \longrightarrow \mathbf{A} \mathbf{b}$, where

$$
\underline{C}_{n}(X ; L)(G / H)=L(G / H) \otimes_{\mathbb{Z} \underline{\pi}_{1}(X)(G / H)} C_{n}\left(\widetilde{X}^{H}\right),
$$

and, for a $G$-map $\widehat{g}: G / H \longrightarrow G / K, \underline{C}_{n}(X ; L)(\widehat{g})=L(\widehat{g}) \otimes C_{n}(\widetilde{g})$. Clearly, these give rise to a chain complex $\underline{C}_{*}(X ; L)$ in the abelian category of abelian $O_{G^{-}}$groups. Then, $\operatorname{Hom}_{L}\left(\underline{C}_{*}(X ; L), M_{0}\right)$ becomes a cochain complex of groups whose $n$th group consists of $L$-invariant natural transformations $\underline{C}_{n}(X ; L) \longrightarrow M_{0}$.

Lemma 2.5. There is an isomorphism

$$
\Psi: S_{\pi, G}^{*}\left(\mathcal{U} ; M_{0}\right) \longrightarrow \operatorname{Hom}_{L}\left(\underline{C}_{*}(X ; L), M_{0}\right)
$$

of cochain complexes.

Proof. Define $\Psi$ and its inverse $\Psi^{\prime}$ in the following way. Let $c=\left\{c_{H}\right\}_{H \subseteq G} \in$ $S_{\pi, G}^{n}\left(\mathcal{U} ; M_{0}\right), T \in \operatorname{Hom}_{L}\left(\underline{C}_{n}(X ; L), M_{0}\right), l \in L(G / H)$, and $\sigma: \triangle_{n} \longrightarrow \widetilde{X}^{H}$ be a singular $n$-simplex. Then, set

$$
\Psi(c)(G / H)(l \otimes \sigma)=\omega(G / H)\left(l, c_{H}(\sigma)\right), \text { and }\left(\Psi^{\prime}(T)\right)_{H}(\sigma)=T(G / H)(1 \otimes \sigma) .
$$

It does not pose any difficulty to verify that $\Psi$ and $\Psi^{\prime}$ are cochain maps inverse to one other (cf. $[8, \S 9])$.

The point to note here is that $G$ has to be finite for $\Psi^{\prime}$ to be well defined.

Proof of Proposition 2.2. The category of abelian $L$-invariant $O_{G}$-groups possesses sufficiently many injectives. Let $M_{0}^{*}$ be an injective resolution of $M_{0}$ in this category. Then, in view of Lemma 2.5, the bicomplex $\operatorname{Hom}_{L}\left(\underline{C}_{*}(X ; L), M_{0}^{*}\right)$ provides a spectral sequence $E(X, L, M)$ in which

$$
E_{2}^{p, q}=\operatorname{Ext}^{p}\left(\underline{H}_{q}(X, L), M_{0}\right) \Longrightarrow H_{G}^{p+q}(X ; M),
$$

where $\underline{H}_{q}(X ; L): O_{G} \longrightarrow \mathbf{A b}$ is given by $\underline{H}_{q}(X ; L)(G / H)=H_{q}\left(X^{H} ; L(G / H)\right)$ which is the ordinary cohomology of $X^{H}$ with local coefficients $L(G / H)$.

Now if $f: X \longrightarrow Y$ is a $G$-map and $M$ is an equivariant local coefficients system on $Y$, then $f$ induces a map of the spectral sequences $f^{*}: E\left(Y, \underline{\pi}_{1}(Y), M\right) \longrightarrow$ $E\left(X, f^{*} \underline{\pi}_{1}(Y), f^{*} M\right)$, where $\underline{\pi}_{1}(Y)$ is as in Example 2.3, and $f^{*} \underline{\pi}_{1}(Y)$ is as in Example 2.4. If $f$ is $G$-acyclic, then $f^{*}$ is an isomorphism at the $E_{2}$-level, by Proposition 2.1 and Proposition (4.3) of [1]. Consequently, $f^{*}: H_{G}^{*}(Y ; M) \longrightarrow$ $H_{G}^{*}\left(X ; f^{*} M\right)$ is an isomorphism. This completes the proof. 


\section{Proof of the theorems}

Proof of Theorem 1.1. It is possible to convert a $G$-space $X$ into an $O_{G \text {-space by }}$ means of a functor $\mathcal{R}$ defined by $\mathcal{R}(X)(G / H)=X^{H}, \mathcal{R}(X)(\widehat{g})=g$ (left translation). Conversely, Elmendorf [5] defined a functor $\mathcal{S}: O_{G}$-spaces $\longrightarrow G$-spaces, and a natural transformation $N: \mathcal{R S} \longrightarrow i d$ such that, for each $O_{G}$-space $T$ and each $H \subseteq G, N(T)(G / H):(\mathcal{S} T)^{H} \longrightarrow T(G / H)$ is a homotopy equivalence. In particular, $N(\mathcal{R}(X))(G /\{e\}): \mathcal{S R} X \longrightarrow X$ is a natural $G$-homotopy equivalence.

Now, if $X$ is a $G$-space, then using the Kan-Thurston theorem [6] for each $X^{H}$, we get a group $\lambda(G / H)$ with a perfect normal subgroup $\eta(G / H)$, and a fibration $p(G / H): K(\lambda(G / H), 1) \longrightarrow X^{H}$ satisfying the conditions that $p(G / H)$ is acyclic, and $\operatorname{Ker} \pi_{1}(p(G / H))=\eta(G / H)$ (note that here we are using $O_{G}$ as an indexing set). By naturality, these fibrations produce an $O_{G}$-fibration $p: E \longrightarrow B$, where $E=\mathcal{R} K(\lambda, 1)$ and $B=\mathcal{R} X$. Applying the Elmendorf's functor $\mathcal{S}$ to it, we get a $G$-map $\mathcal{S} p: \mathcal{S} E \longrightarrow \mathcal{S} B$ so that $(\mathcal{S} E)^{H}$ and $(\mathcal{S} B)^{H}$ have the homotopy types of $K(\lambda(G / H), 1)$ and $X^{H}$ respectively. This gives Theorem 1.1 immediately.

Proof of Theorem 1.2. First note that the plus construction $W \longrightarrow W_{P}^{+}$, where $W$ is a $\mathrm{CW}$-space and $P$ is a perfect normal subgroup of $\pi_{1}(W)$, is not functorial, but functorial up to homotopy. However, it is possible to choose $W_{P}^{+}$from its homotopy type so that $W \longrightarrow W_{P}^{+}$becomes functorial. This may be done in the following way. Let $\alpha: \widetilde{W}_{P} \longrightarrow W$ be the covering space of $W$ corresponding to the subgroup $P$ so that $\operatorname{Im} \pi_{1}(\alpha)=P$, and let $\beta: A\left(\widetilde{W}_{P}\right) \longrightarrow \widetilde{W}_{P}$ be the natural fibration obtained by applying the acyclic functor $A$ of Dror [4]. Then the cofibre $i: W \longrightarrow C_{\alpha}$ of $\alpha \circ \beta: A\left(\widetilde{W}_{P}\right) \longrightarrow W$, where $C_{a}$ is the mapping cone of $\alpha$, is homotopically equivalent to $W \longrightarrow W_{P}^{+}$(over $W$ ). These cofibres provide a functor which may be called the functorial plus construction.

Now if $X$ is a $G$-space and $\eta$ is a perfect normal $O_{G}$-subgroup of $\underline{\pi}_{1}(X)$, then applying the functorial plus construction to each $X^{H}$ we get an acyclic map $f(G / H)$ : $X^{H} \longrightarrow\left(X^{H}\right)_{\eta(G / H)}^{+}$such that $\operatorname{Ker} \pi_{1}(f(G / H))=\eta(G / H)$. These maps give a morphism of $O_{G^{-}}$spaces which turns into a $G$-map $f^{\prime}: \mathcal{S R} X \longrightarrow X_{\eta}^{+}$by means of the Elmendorf's functor $\mathcal{S}$. Then a composition of a $G$-homotopy equivalence $X \longrightarrow \mathcal{S R} X$ with $f^{\prime}$ gives the required $G$-acyclic map $f: X \longrightarrow X_{\eta}^{+}$. This completes the proof of Theorem 1.2.

Proof of Theorem 1.3. If $h$ exists, then $\underline{\pi}_{1}\left(f^{\prime}\right)=\underline{\pi}_{1}(h) \circ \underline{\pi}_{1}(f)$, and therefore $\operatorname{Ker} \underline{\pi}_{1}(f) \subseteq \operatorname{Ker} \underline{\pi}_{1}\left(f^{\prime}\right)$. Conversely, consider the $G$-push out diagram, and its restriction to each $H$-fixed point set
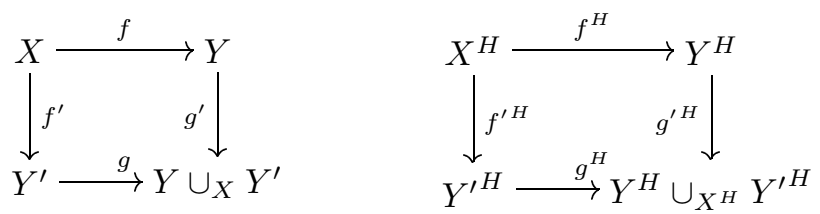

The second diagram implies that $g^{H}$ is acyclic, since $f^{H}$ is so, and, by the van Kampen theorem, $\pi_{1}\left(g^{H}\right)$ is an isomorphism, since $\operatorname{Ker} \pi_{1}\left(f^{H}\right) \subseteq \operatorname{Ker} \pi_{1}\left(f^{\prime H}\right)$. Therefore $g^{H}$ is a homotopy equivalence, and hence $g$ is a $G$-homotopy equivalence, by the equivariant Whitehead theorem [3, p. 107]. Then, if $g_{1}$ is a $G$-homotopy inverse of $g, h=g_{1} \circ g^{\prime}: Y \longrightarrow Y^{\prime}$ is the required $G$-map with $h \circ f \simeq_{G} f^{\prime}$. 
Clearly $h$ is $G$-acyclic if $f^{\prime}$ is so, and, since $\underline{\pi}_{1}(h)$ is an isomorphism if and only if $\operatorname{Ker} \underline{\pi}_{1}(f)=\operatorname{Ker} \underline{\pi}_{1}\left(f^{\prime}\right)$, the last assertion follows.

To see that $h$ is unique up to $G$-homotopy equivalence, suppose that $j: F \longrightarrow X$ is the $G$-homotopy fibre of $f: X \longrightarrow Y$. Then, since $f \circ j \simeq_{G} y^{0}, f$ extends to a $G$-map $k: X \cup_{j} C F \longrightarrow Y$ over the equivariant mapping cone of $j$. The $G$-map $k$ is actually a $G$-homotopy equivalence, because its restriction to each $H$-fixed point set $k^{H}: X^{H} \cup C F^{H} \longrightarrow Y^{H}$ is acyclic and $\pi_{1}\left(k^{H}\right)$ is an isomorphism. Thus we have an equivariant coexact sequence

$$
F \longrightarrow X \longrightarrow Y \longrightarrow \Sigma F
$$

where $\Sigma F$ is the equivariant suspension of $F$. Since $\Sigma F^{H}$ is simply connected and $\widetilde{H}_{*}\left(\Sigma F^{H} ; \mathbb{Z}\right)=0, \Sigma F^{H}$ is contractible. This implies that $\Sigma F$ is $G$-contractible by the equivariant Whitehead theorem. Thus the map $f^{*}:\left[Y, Y^{\prime}\right]_{G}^{0} \longrightarrow\left[X, Y^{\prime}\right]_{G}^{0}$ in the equivariant Barratt-Puppe sequence $\left[3\right.$, p. 142] is injective, where $\left[Y, Y^{\prime}\right]_{G}^{0}$ denotes the set of base point preserving $G$-homotopy classes of $G$-maps $Y \longrightarrow Y^{\prime}$. This ensures the uniqueness of $h$, and the proof of Theorem 1.3 is complete.

The assertion of Theorem 1.4 is now straightforward.

In conclusion, we remark that the proofs appearing in this section remain valid if $G$ is a compact Lie group and $X$ is a $G$-CW-space with each $X^{H}$ a connected $\mathrm{CW}$-space.

\section{ACKNOWLEDGEMENT}

We are grateful to the referees and Dr. Goutam Mukherjee for helpful comments.

\section{REFERENCES}

1. A. J. Berrick, An approach to algebraic K-theory, Research Notes in Math. 56 (Pitman, 1982). MR 84g: 18028

2. G. E. Bredon, Equivariant cohomology theories, Lecture Notes in Math. 34 (Springer-Verlag, 1967). MR 35:4914

3. T. tom Dieck, Transformation groups, de Gruyter Studies in Math. 8 (Walter de Gruyter, Berlin, New York, 1987). MR 89c:57048

4. E. Dror, Acyclic spaces, Topology 11 (1972), 339-348. MR 47:4262

5. A. D. Elmendorf, System of fixed point sets, Trans. Amer. Math. Soc. 277 (1983), 275-284. MR 84f:57029

6. D. M. Kan and W. P. Thurston, Every connected space has the homology of a $K(\pi, 1)$, Topology 15 (1976), 253-258. MR 54:1210

7. S. Illman, Equivariant singular homology and cohomology, Mem. Amer. Math. Soc. 19 (1975). MR 51:11482

8. A. Mukherjee and G. Mukherjee, Bredon-Illman cohomology with local coefficients, Quart. J. Math. Oxford(2) 47 (1996), 199-219. CMP 96:15

9. D. G. Quillen, Cohomology of groups, Actes Congrès Int. Math. Nice, T. 2 (1970), 47-51. MR 58:7627a

Stat-Math Division, Indian Statistical Institute, 203 B. T. Road, Calcutta 700 035, INDIA

E-mail address: amiya@isical.ernet.in

School of Mathematics, SpiC Science Foundation, 92, G. N. Chetty Road, Madras 600 017, INDIA

E-mail address: anirudha@ssf.ernet.in 